\title{
Pharyngeal carriage rates of Neisseria meningitidis in health care professionals at a tertiary university pediatric hospital
}

\author{
Lisa-Maria Steurer ${ }^{1} \cdot$ Mathias Hetzmannseder ${ }^{1} \cdot$ Birgit Willinger $^{2} \cdot$ Peter Starzengruber $^{2} \cdot$ Claudia Mikula $^{3}$. \\ Andrea Kormann-Klement ${ }^{3} \cdot$ Michael Weber $^{4} \cdot$ Angelika Berger $^{1} \cdot$ Agnes Grill $^{1}$
}

Received: 2 January 2020 / Accepted: 1 April 2020 / Published online: 24 April 2020

(C) The Author(s) 2020

\begin{abstract}
Pharyngeal carriage is the reservoir for Neisseria meningitidis in the population and the first step in disease transmission. Especially in young infants and adolescents, $N$. meningitidis can cause serious invasive infection with high fatality rates and high rates of long-term sequelae among survivors. The aim of this study was to determine $N$. meningitidis colonization rates in asymptomatic health care professionals at a tertiary university pediatric hospital and to identify risk factors for carriage. This cross-sectional meningococcal carriage survey was conducted between April and October 2018 at the Medical University of Vienna. Individuals working as nurses, pediatricians, or medical students were enrolled. Oropharyngeal swabs were directly plated onto selective agar plates and conventional culture was used for bacterial identification. Meningococcal isolates were further characterized using whole-genome sequencing. A total of 437 oropharyngeal specimens were collected. Overall, meningococcal carriage prevalence was $1.14 \%$ (5/437), with $0.7 \%$ (3/437) for capsular genotype B, and $0.5 \%$ (2/437) for capsular genotype W. Mean age of carriers was significantly lower than of non-carriers ( $24.2 \mathrm{vs.} 35.8 ; p=0.004)$. The highest carriage rate of 4.4\% (4/91) was found in the age group 18-25. Carriage was negatively associated with age and timespan working in pediatrics. This is the first study evaluating the prevalence of Neisseria meningitidis carriage in health care professionals working in Pediatrics and Adolescent Medicine. Carriage was in general lower than expected for all age groups, implicating a low risk of meningococcal transmission via this population.
\end{abstract}

Keywords Neisseria meningitidis $\cdot$ Pharyngeal carriage rates $\cdot$ Pediatric health care professionals $\cdot$ Meningococcal carriage $\cdot$ Pediatric hospital

\section{Introduction}

Neisseria meningitidis is a diplococcal, aerobic, Gram-negative, and obligate human bacterium categorized into 12 different serotypes according to capsular polysaccharide structure.

Lisa-Maria Steurer

lisa-maria.steurer@meduniwien.ac.at

1 Department of Pediatrics and Adolescent Medicine, Division of Neonatology, Pediatric Intensive Care Medicine and Neuropediatrics, Medical University of Vienna, Vienna, Austria

2 Department of Laboratory Medicine, Division of Clinical Microbiology, Medical University of Vienna, Vienna, Austria

3 Institute for Medical Microbiology and Hygiene, Austrian Agency for Health and Food Safety, Graz, Austria

4 Section for Medical Statistics, CeMSIIS, Medical University of Vienna, Vienna, Austria
The epidemiologically most relevant serogroups are A, B, C, $\mathrm{W}$, and $\mathrm{Y}$ accounting for $>90 \%$ of invasive meningococcal disease (IMD) cases worldwide [1].

It can colonize the human nasopharynx as part of its complex microbiota without affecting the host. Carriage is a highly variable phenomenon and differs with age, region, and setting [2]. Lowest carriage rates were found in newborns and infants, then it increases throughout childhood, peaking in adolescence and young adult age, before gradually declining again in older age [3]. In closed or semi-closed populations such as military recruits and university students living in campuses, or in disease outbreak settings, it can reach up to $25 \%$ or even more [4].

As the human nasopharynx is the unique biological niche for $N$. meningitidis, the carrier state is regarded as the reservoir for meningococcal transmission being passed from one person to the other via respiratory droplets, direct contacts with IMD patients, or more often asymptomatic carriers. 
Acquisition usually results in asymptomatic carriage, but in rare cases, the bacteria can escape the mucosal barrier and invade the bloodstream leading to lifethreatening conditions such as meningitis and sepsis with letality rates of $10-15 \%$ and resulting in severe long-term sequelae in up to $20 \%$ of survivors [5]. Hence, it remains an important public health issue globally, despite a generally low overall IMD incidence of 0.6 annual cases per 100,000 inhabitants across Europe [6].

Highest disease incidences have consistently been found in the pediatric patient cohort, i.e., in infants in the first year of life when maternal antibodies progressively fade out, in 1-4year-old children, and in adolescents [7]. Infants are regarded most susceptible to severe IMD due to their immature immune system, whereas adolescents are at risk because of high carriage and transmission rates.

An elevated risk for meningococcal infection and disease has also been described for health care workers exposed to patients with meningococcal disease. The excess risk for physicians, nurses, or paramedics with intensive close contact during airway management or mouth-to-mouth resuscitation of infected cases is estimated 25 times that in the general population [8]. The majority of occupationally acquired meningococcal disease, however, occurred in the setting of improper precaution, i.e., unprotected exposure to infected patients without use of post-exposure chemoprophylaxis $[9,10]$.

Carriage studies are important to understand the epidemiology and pathogenesis of meningococcal disease and for establishing vaccination strategies, since vaccination of population cohorts with highest carriage rates has been proven most effective to confer herd protection [11].

This is especially important in medical staff working in pediatrics treating the patient cohort the most susceptible and vulnerable to severe meningococcal disease. Currently, there is no surveillance of asymptomatic carriers in this population.

In this cross-sectional carriage study, we aimed to assess meningococcal carriage rates in a health care personnel cohort working in pediatrics and adolescent medicine where there is permanent contact to patient cohorts with the highest risk for meningococcal carriage and disease.

\section{Materials and methods}

\section{Study design and participants}

This study was conducted as a cross-sectional carriage survey evaluating Neisseria meningitidis colonization in asymptomatic health care professionals at the department of pediatrics and adolescent medicine of the Medical University of Vienna during a 6-month study period from 20 April-30 October 2018. All medical staff members working as nurses or doctors on 9 different pediatric wards, as well as medical students passing an internship for at least 4 weeks, were recruited for participation on voluntary basis. Exclusion criteria were antibiotic therapy in the preceding 4 weeks and working less than 4 weeks in pediatrics. Although not assessed formally, approximately $10-20 \%$ of eligible individuals refused participation.

\section{Specimen and data collection}

Prior to sample collection, each volunteer signed a written informed consent form and was administered a de-identified self-administered questionnaire consisting of sociodemographic data, e.g., sex, age, profession, time span working in pediatrics, main work setting (neonatology, pediatric intensive care unit, outpatient department, general pediatric unit), and information on potential risk factors associated with meningococcal carriage, such as active smoking (defined as smoking at least one cigarette daily), recent symptoms of upper respiratory tract infection in the last 2 weeks, number of household members, number and age of children/adolescents living in the same household, and recent or past contacts with a patient with IMD [12-14]. Furthermore, the questionnaire collected the meningococcal vaccination status of participants.

Oropharyngeal samples were collected by trained personnel using a sterile flocked nylon swab (FLOQSwabs/ ESwab $^{\mathrm{TM}}$, Copan Diagnostics Inc., Brescia, Italy). One single swab was taken from the posterior wall of the oropharynx behind the uvula via the mouth of each subject using a standardized technique [15] and was immediately plated onto a selective agar medium (Chocolate agar + PolyViteX VCAT3, described by Thayer \& Martin, bioMérieuxSA, Marcy l'Étoile, France) and on Columbia Blood Agar.

\section{Laboratory procedures and bacterial identification}

The collected plates were processed at the microbiological laboratory of the Medical University of Vienna, where the media were incubated within $3 \mathrm{~h}$ at $37{ }^{\circ} \mathrm{C}$ in $5 \% \mathrm{CO}_{2}$ and examined at 18-24 h and at $48 \mathrm{~h}$ for the growth of $N$. meningitidis. All colonies suspected for being $N$. meningitidis underwent gramstaining and additional identification with matrix-assisted laser desorption/ionization time-of-flight mass spectrometry analysis (MALDI-Biotyper, Bruker, Germany) according to the manufacturer's instructions.

All confirmed isolates were sent to the Austrian National Reference Center for Meningococcal Disease in Graz (AGES), where identification of serogroups was performed using slide agglutination and whole-genome sequencing (WGS). The WGS was performed on purified genomic DNA by parallel high-throughput next-generation sequencing (NGS) technologies on Illumina MiSeq platform (Illumina Inc., San Diego, CA, USA) according to the manufacturer's protocol. 
Further analysis concerning sequence types, clonal complex, and outer membrane (PorA and FetA) variants and alleles of the meningococcal isolates was performed using the Neisseria species MLST database (https://pubmlst.org/ neisseria) of the University of Oxford.

\section{Statistical analysis}

All computations were performed using IBM SPSS Statistics for Windows version 23.0 (IBM, Armonk, NY).

Nominal data are presented using percentages as well as absolute frequencies. Metric data are presented using mean \pm standard deviation. For the continous variable "age," also median, interquartile range (IQR), and total range were determined; additionally, age was recoded into four age groups (18-25, 26-35, 36-45, > 45). Differences between the groups given categorical dependent variables were compared using two-sided Fisher's exact test or chi2 test as appropriate [16]. In addition, crosstabs and Fisher exact tests were used to assess the correlation between risk factors and carriage rates. $p$ values equal or below $5 \%$ were considered statistically significant.

Ethics statement The study protocol, assent form, and the questionnaire were approved by the ethics committee at the Medical University of Vienna (2018-01-21, reg.-no. 2229/2017). Each participant signed written informed consent. Additional approval was obtained from the data protection committee and the employee organization of the Medical University Vienna.

\section{Results}

\section{Participant characteristics and carriage prevalence}

A total of 484 oropharyngeal samples were collected, of which 47 subjects had to be excluded according to our study protocol. Of them, 38 were working in pediatrics for less than 4 weeks, eight had antibiotic treatment in the preceding 4 weeks, and one had to be excluded due to a missing questionnaire and assent form.

The remaining 437 participants were included for further analysis and comprised 307 nurses, 110 doctors, and 20 medical students; $85.6 \%$ (374/437) were females and 14.4\% (63/ 437) were males.

The mean age of our study population was 35.6 years (SD 10.7, median 33, IQR 17, total range 18-64 years); 34.9 years (SD 10.6) for nurses, 39.2 years (SD 10.3) for doctors, and 26.9 years (SD 3.5) for medical students.

The majority were non-smokers $(85.4 \%, 373 / 437)$.

According to the questionnaire, $22.4 \%$ (98/437) had experienced symptoms of respiratory tract infection within 2 weeks prior to swabbing. Eight percent (35/437) reported contact to a patient with invasive meningococcal disease within 1 year prior to sampling.

Full demographic characteristics of the participants are shown in Table 1.

Data on meningococcal vaccination status were available for $94 \%$ (411/437) of our study population and showed an overall vaccination rate of $28.5 \%$ (117/411). The meningococcal vaccination rate among nurses was $17.5 \%(50 / 286)$, among doctors 53.3\% (57/105), and 55.6\% (10/18) among medical students. Characteristics of participants' meningococcal vaccination history are shown in Table 1 .

Among the 437 pharyngeal samples, five $N$. meningitidis isolates were recovered, rendering an overall carriage rate of $1.14 \% ; 0 \%$ in doctors $(0 / 110), 1.3 \%$ in nurses $(4 / 307)$, and $5 \%$ in medical students $(1 / 20)$. Carriage prevalence was highest among the age group $18-25$ years $(4.4 \%, 4 / 91)$. None of the carriers was older than 35 years and none of them had any vaccination against meningococci.

Serogroup identification showed 3 serogroup B and 2 serogroup $\mathrm{W}$ isolates, accordingly $0.69 \%$ and $0.46 \%$ of the study population. We did not find other serogroups or unencapsulated meningococci.

Genotypic characteristics of recovered N. meningitidis isolates are shown in Table 2.

\section{Association with N. meningitidis carriage}

$N$. meningitidis carriers were significantly younger than noncarriers ( 24.2 vs. $35.8 ; p=0.004)$.

Univariate analysis revealed that age and the timespan working in pediatrics were significantly inversely associated to meningococcal carriage. In contrast, there was no statistically significant association between carriage and recent contact to an IMD patient, respiratory tract infection, smoking, and vaccination against any meningococcal serogroup. No significant differences in carriage were found between different professions and main work settings. In addition, no differences in detection rate could be found according to the month of swab collection. The number of participants with carriage $(n=5)$ was too small to perform a multivariable model or to assess an interaction effect between variables.

\section{Discussion}

To our knowledge, this is the first study evaluating $N$. meningitidis carriage rates in asymptomatic health personnel working in pediatrics and adolescent medicine. In addition, sparse data are available for health care workers in general.

The main finding was a low overall meningococcal carriage prevalence of $1.14 \%$. This low prevalence is possibly related to the characteristics of our study population, showing a low-risk profile according to previously reported risk factors 
Table 1 Participant characteristics: demographic characteristics of total sample $(n=437)$ and N. meningitidis carriers $(n=5)$

\begin{tabular}{|c|c|c|c|}
\hline Category & & Overall participants $n(\%)$ & Carriers $n(\%)$ \\
\hline Total number & & $437(100 \%)$ & $5 / 437(1.14 \%)$ \\
\hline \multirow[t]{3}{*}{ Profession } & Nurses & $307(70.3 \%)$ & $4 / 307(1.3 \%)$ \\
\hline & Doctors & $110(25.2 \%)$ & $0 / 110(0 \%)$ \\
\hline & Medical Students & $20(4.5 \%)$ & $1 / 20(5 \%)$ \\
\hline \multirow[t]{4}{*}{ Age group (years) } & $18-25$ & $91(20.8 \%)$ & 4/91 (4.4\%) \\
\hline & $26-35$ & $169(38.7 \%)$ & $1 / 169(0.6 \%)$ \\
\hline & $36-45$ & $89(20.4 \%)$ & 0 \\
\hline & $>45$ & $88(20.1)$ & 0 \\
\hline \multirow[t]{4}{*}{ Mean age (years) } & Total & $35.6(10.7 \mathrm{SD})$, median 33 & 24.2 (4.6 SD), median 23 \\
\hline & Nurses & $34.9(10.6 \mathrm{SD})$ median 32 & - \\
\hline & Doctors & $39.2(10.3 \mathrm{SD})$ median 36 & - \\
\hline & Medical Students & $26.9(3.5 \mathrm{SD})$ median 27 & - \\
\hline \multirow[t]{4}{*}{ Timespan working in pediatrics (years) } & $<1$ & $86(19.7 \%)$ & $4 / 86(4.7 \%)$ \\
\hline & $1-5$ & $127(29.1 \%)$ & $1 / 127(0.8 \%)$ \\
\hline & $6-10$ & $60(13.7 \%)$ & $1 / 60(1.7 \%)$ \\
\hline & $>10$ & $164(37.5 \%)$ & 0 \\
\hline \multirow[t]{2}{*}{ Gender } & Female & $374(85.6 \%)$ & $4 / 374(1.1 \%)$ \\
\hline & Male & $63(14.4 \%)$ & $1 / 63(1.6 \%)$ \\
\hline Active Smokers & & $64(14.6 \%)$ & $1 / 64(1.6 \%)$ \\
\hline Resp tract infections ( $<2$ weeks) & & $98(22.4 \%)$ & 0 \\
\hline Mean household size (persons) & & $2.6(1.2 \mathrm{SD})$, median $2.0(1-8)$ & $2.4(1.1 \mathrm{SD})$, median $2.0(1-4)$ \\
\hline Recent IMD contact ( $<1$ year) & & $39(8.9 \%)$ & 0 \\
\hline \multirow{4}{*}{$\begin{array}{l}\text { Vaccination against } N \text {. meningitidis } \\
\quad \text { (any serogroup) }\end{array}$} & Total sample & $117 / 411(28.5 \%)$ & 0 \\
\hline & Nurses & $50 / 286(17.5 \%)$ & 0 \\
\hline & Doctors & $57 / 107(53.3 \%)$ & 0 \\
\hline & Medical Students & $10 / 18(55.6 \%)$ & 0 \\
\hline \multirow[t]{4}{*}{ Vaccination against serogroup ACWY } & Total sample & $66 / 415(15.9 \%)$ & 0 \\
\hline & Nurses & $21 / 289(7.3 \%)$ & 0 \\
\hline & Doctors & $39 / 108(36.1 \%)$ & 0 \\
\hline & Medical Students & $6 / 18(33.3 \%)$ & 0 \\
\hline \multirow[t]{4}{*}{ Vaccination against serogroup B } & Total sample & $50 / 415(12.0 \%)$ & 0 \\
\hline & Nurses & $9 / 289(3.1 \%)$ & 0 \\
\hline & Doctors & $34 / 108(31.4 \%)$ & 0 \\
\hline & Medical Students & $7 / 18(38 \%)$ & 0 \\
\hline
\end{tabular}

associated with meningococcal carriage: advanced age of study participants, low number of smokers, $85 \%$ of the study population being female, and high socioeconomic status [1]. Active smoking is a well-recognized risk factor for meningococcal carriage, since it causes structural changes in the respiratory tract mucosa and impairs immune response $[17,18]$. Male gender is associated with significantly higher carriage rates in most carriage studies [19].
Table 2 Molecular characterization of the five recovered $N$. meningitidis isolates: genogroup, sequence types, clonal complex, and outer membrane variants (PorA and FetA)

\begin{tabular}{lllllll}
\hline Serogroup & Capsule group & ST & Clonal complex & PorA VR1 & PorA VR2 & FetA VR \\
\hline B & B & 213 & ST-213 complex & 22 & 14 & F5-5 \\
W & W & 11 & ST-11 complex & 5 & 2 & F1-1 \\
W & W & 10,076 & ST-35 complex & $22-1$ & $14-40$ & F3-9 \\
B & B & 1572 & & 18 & 25 & F1-7 \\
B & B & 9661 & ST-213 complex & 22 & 14 & F5-5 \\
\hline
\end{tabular}


However, we believe that the most important aspect in our cohort is the relatively high mean age of participants being 35.6 years compared to most meningococcal carriage studies. Even the mean age of students was 26.9 years. The carriage prevalence found among individuals aged 18-25 years, who are more often the target group in meningococcal carriage studies, was $4.4 \%$ (4/91) in our cohort and hence significantly higher than in older age groups. This finding is well in line with the literature, as highest carriage rates have consistently been found in this age group [3]. This effect seems to be most likely due to a convergence of risk factors and social habits that facilitate close interpersonal contacts observed in young adults than age per se $[20,21]$. Studies comparing carriage rates of young adults at entry of university or military service to those later in the academic year or at the end of military service observed much lower carriage prevalence at the beginning of university or military service [22,23]. For example, a carriage survey of military recruits aged 18-24 years in Finland showed a low overall meningococcal carriage rate of $2.2 \%$ (20/892) among those beginning military service, compared to $18.5 \%(151 / 818)$ at the end of their military service [24].

Most meningococcal carriage studies have been undertaken in at-risk populations, i.e., teenagers, university students, military recruits, disease outbreak settings, and Hajj pilgrims [2, 3, 19]. N. meningitidis carriage in older individuals, i.e., beyond the teenage years and young adult age, especially in low-risk settings, is poorly understood, primarily due to a dearth of carriage data available and hence meningococcal carriage in the total population might have been overestimated.

A recent carriage survey among individuals aged 65 years and older in Germany found a remarkably low prevalence of colonization with $N$. meningitidis of $0.3 \%$ (2/677; $95 \%$ CI 0 $1.1 \%)$ [25]. These data suggest that carriage rates might dramatically drop in older age groups to a much bigger extent than previously thought. A cross-sectional study in Tuscany, Italy, found a carriage prevalence of $4.8 \%$ in individuals aged 11-45 years presenting at immunization clinics. Among the age group 31-45 years, the carriage prevalence was 2.4\% (20/ 828 ), while it was $9 \%$ (37/434) in the age group 20-30 years [26]. A study conducted in 2014 in Hajj pilgrims with a mean age of 50 years found $1.3 \%$ meningococcal carriers in individuals originating from low endemic countries, while $8.9 \%$ of pilgrims from high endemic regions (sub-Saharan meningitis belt) were tested positive for $N$. meningitidis [27].

Other recent studies also described lower meningococcal carriage prevalence than anticipated even in high-risk settings. In a carriage survey in Greece in 2014/2015 including 1420 individuals aged 18-26 years, Tryfinopoulou et al. observed significantly lower carriage prevalences compared to their previous studies 20 years ago among military recruits $(15 \%$ vs. $25 \%, p<0.05)$ and university students $(10.4 \%$ vs $18 \%, p=0.002)$ [28].

Carriage rates in university students aged $17-25$ years in South Australia were $6.3 \%$ and hence lower than anticipated
[29]. Authors conclude that possible factors for lower meningococcal carriage rates might be a reduction to smoking habits and meningococcal vaccination.

Significant impact on carriage rates has been reported for most meningococcal conjugate vaccines [30-32]. In Austria, meningococcal vaccination is recommended for personnel working in pediatric facilities because of an elevated risk of exposure to IMD cases [33]. In our subcohort of doctors, there was a high meningococcal vaccine coverage of more than $50 \%$; in this subcohort, we did not find any $N$. meningitidis carrier, while none of the detected carriers in our study population had any vaccination against meningococci. However, this effect was not statistically significant.

Meningococcal carriage prevalence among medical staff members in general has not largely been investigated, and information concerning this cohort is limited to isolated reports. Coch Goia et al. tested 200 staff members in a university hospital in Brazil. Carriage prevalence in the hospital employee cohort (mean age 41.9 ) was $3 \%$ (3/100), while it was $15 \%$ $(15 / 100)$ in students (mean age 23.6). These findings again suggest age as the predominant factor for carriage [34].

The estimated relative risk concerning work-related IMD is increased for health care providers exposed to respiratory droplets of affected patients compared to the general population [8]. The majority of published meningococcal infections in health care workers, however, occurred in the setting of improper precautionary measures. Using appropriate personal protective equipment of any kind and post-exposure chemoprophylaxis, the risk for occupational meningococcal transmission resulting in either secondary meningococcal disease or carriage is estimated to be low $[9,10]$.

Despite all aforementioned aspects, our detected carriage rates are still less than anticipated even for the youngest age group investigated compared to most of the pre-existing literature. Key limitation in interpreting the presented results is that a comparable cohort of pediatric clinic employees has not yet been investigated.

Factors that might hypothetically be special in a pediatric health personnel cohort include frequent contact to IMD cases with subsequent post-exposure prophylaxis that also effectively eradicates colonization with any meningococci [35]. In addition, unknown factors of social behavior patterns in this cohort might contribute.

This is not only the first carriage study in a pediatric health personnel setting but also the first meningococcal carriage study in Austria. The incidence of IMD in Austria is among the lowest in Europe. There has been a gradual decline over the past 10 years; in 2018, the IMD incidence was 0.34 per 100,000 inhabitants [36]. This low national disease incidence might be an additional explanation for the low carriage prevalence found, as it is assumed to find higher carriage rates in high endemic countries [27]. 
Sample collection from April to October without collecting swabs during winter should not have had an impact on carriage rate, especially considering that the mean duration of meningococcal carriage is estimated to range from 3.4 to 11.7 months $[37,38]$. Unlike meningococcal disease, carriage does not seem to be relevantly influenced by seasonality according to the literature $[2,13]$.

In terms of serogroup distribution, we found serogroup B and serogroup W in carriers. Serogroup B is usually the dominant capsular group in carriage studies across Europe and in most countries worldwide, while serogroup $\mathrm{W}$ was historically less frequently detected in carriage studies since those strains seem to have a shorter duration of carriage [2, 17]. However, during the last years, an increase in MenW IMD was observed in most European countries, following emerging MenW IMD cases in South America since 2004 and hence $\mathrm{MenW}$ is detected more frequently among carriers [39]. In a recent carriage study in Turkey, MenW accounted for 66.6\% of all recovered meningococcal isolates [21].

Interestingly, we did not observe any non-groupable meningococci, although non-encapsulated $N$. meningitidis are frequently found among carriers according to the literature [26, 40-42].

This cross-sectional carriage survey has several limitations. First, using one single swab might have underestimated carriage rates, since the sensitivity of swabbing is estimated to be 60-83\% [43]. Out of respect for our volunteers and to keep attrition rates low, we decided to take only one pharyngeal swab. In addition, our study was substantially limited by the unexpected small number of carriers, so that our analysis did not have enough power to assess significance of potential associations or risk factors for carriage. Further limitations were the lack of a control group and of longitudinal data.

Methodically, we decided for direct plating of swabs followed by conventional culture methods for bacterial identification, although recently reported methods using PCR after THB (ToddHewitt broth) culture might have had a higher yield [44]. However, this is not standard yet. Conventional culture has been used in the majority of carriage studies, which allows better comparison of results but might bear the risk of underestimating the true level of carriage $[2,3]$. With the possibility of collecting swabs in a university hospital setting with immediate availability of microbiological processing of collected plates, we were able to avoid altering results due to transport delays.

Despite aforementioned limitations, our study provides important new aspects concerning the epidemiology of $N$. meningitidis in pediatric hospitals. Our results show that the prevalence of pharyngeal meningococcal colonization is low among health care personnel working in pediatrics and adolescent medicine, and thus, the risk of meningococcal transmission is low.

Acknowledgments The authors would like to thank all volunteers for participating in the study.
Funding information Open access funding provided by Medical University of Vienna. This work was financially supported by Pfizer Vaccine Research and Development. The funder is independent of study management and analysis of the data. Pfizer was provided the opportunity to review a preliminary version of the manuscript for factual accuracy but the authors are solely responsible for final content and interpretation. The authors received no financial support or other form of compensation related to the development of the manuscript.

\section{Compliance with ethical standards}

Conflict of interest The authors declare that they have no conflict of interest.

Ethical approval The study protocol, assent form, and the questionnaire were approved by the ethics committee at the Medical University of Vienna (2018-01-21, reg.-no. 2229/2017). Additional approval was obtained from the data protection committee and the employee organization of the Medical University Vienna.

Informed consent Written informed consent was signed by each participant.

Open Access This article is licensed under a Creative Commons Attribution 4.0 International License, which permits use, sharing, adaptation, distribution and reproduction in any medium or format, as long as you give appropriate credit to the original author(s) and the source, provide a link to the Creative Commons licence, and indicate if changes were made. The images or other third party material in this article are included in the article's Creative Commons licence, unless indicated otherwise in a credit line to the material. If material is not included in the article's Creative Commons licence and your intended use is not permitted by statutory regulation or exceeds the permitted use, you will need to obtain permission directly from the copyright holder. To view a copy of this licence, visit http://creativecommons.org/licenses/by/4.0/.

\section{References}

1. Yazdankhah SP, Caugant DA (2004) Neisseria meningitidis: an overview of the carriage state. J Med Microbiol 53:821-832

2. Soriano-Gabarró M, Wolter J, Hogea C, Vyse A (2011) Carriage of Neisseria meningitidis in Europe: a review of studies undertaken in the region. Expert Rev Anti-Infect Ther 9:761-774

3. Christensen H, May M, Bowen L, Hickman M, Trotter CL (2010) Meningococcal carriage by age: a systematic review and meta-analysis. Lancet Infect Dis 10:853-861

4. Stephens DS, Greenwood B, Brandtzaeg P (2007) Epidemic meningitis, meningococcaemia, and Neisseria meningitidis. Lancet 369: 2196-2210

5. Pace D, Pollard AJ (2012) Meningococcal disease: clinical presentation and sequelae. Vaccine 30(Suppl. 2):B3-B9

6. European Center for Disease Prevention and Control (2019) Invasive meningococcal disease. Annual epidemiological report for 2017. https://www.ecdc.europa.eu/sites/portal/files/documents/AER for 2017-invasive-meningococcal-disease.pdf. Accessed 22 Oct 2019

7. Dwillow R, Fanella S (2015) Invasive meningococcal disease in the 21st century- an update for the clinician 2015. Curr Neurol Neurosci Rep 15:2

8. Gilmore A, Stuart J, Andrews N (2000) Risk of secondary meningococcal disease in health-care workers. Lancet 356:1654-1655

9. Riccò M, Vezzosi L, Odone A, Signorelli C (2017) Invasive meningococcal disease on the workplaces: a systematic review. Acta Biomed 88:337-351 
10. Pollard AJ, Begg N (1999) Meningococcal disease and healthcare workers. BMJ 319:1147-1148

11. Bijlsma MW, Brouwer MC, Spanjaard L, van de Beek D, van der Ende A (2014) A decade of herd protection after introduction of meningococcal serogroup $\mathrm{C}$ conjugate vaccination. Clin Infect Dis 59:1216-1221

12. Fontanals D, Van Esso D, Pons I, Pineda V, Sanfeliu I, Mariscal D et al (1996) Asymptomatic carriage of Neisseria meningitidis in a randomly sampled population. Serogroup, serotype and subtype distribution and associated risk factors. Clin Microbiol Infect 2: 145-146

13. Caugant DA, Maiden MC (2009) Meningococcal carriage and disease-population biology and evolution. Vaccine 27(Suppl. 2): B64-B70

14. Bruce MG, Rosenstein NE, Capparella JM, Shutt KA, Perkins BA, Collins M (2001) Risk factors for meningococcal disease in college students. JAMA 286:688-693

15. Roberts J, Greenwood B, Stuart J (2009) Sampling methods to detect carriage of Neisseria meningitidis; literature review. J Inf Secur 58:103-107

16. Bewick V, Cheek L, Ball J (2004) Statistics review 8: qualitative data - tests of association. Crit Care 8:46-53

17. Caugant DA, Tzanakaki G, Kriz P (2007) Lessons from meningococcal carriage studies. FEMS Microbiol Rev 31:52-63

18. Imrey PB, Jackson LA, Ludwinsky PH, England AC 3rd, Fella GA, Fox BC et al (1995) Meningococcal carriage, alcohol consumption, and campus bar patronage in a serogroup $\mathrm{C}$ meningococcal disease outbreak. J Clin Microbiol 33:3133-3137

19. Peterson ME, Mile R, Li Y, Nair H, Kyaw MH (2018) Meningococcal carriage in high-risk settings: a systematic review. Int J Infect Dis 73:109-117

20. MacLennan J, Kafatos G, Neal K, Andrews N, Cameron JC, Roberts R et al (2006) Social behavior and meningococcal carriage in British teenagers. Emerg Infect Dis 12:950-957

21. Tekin RT, Dinleyici EC, Ceyhan M, Karbuz A, Salman N, Sutçu M et al (2017) The prevalence, serogroup distribution and risk factors of meningococcal carriage in adolescents and young adults in Turkey. Hum Vacc Immunother 13:1182-1189

22. Jordens JZ, Williams JN, Jones GR, Heckels JE (2002) Detection of meningococcal carriage by culture and PCR of throat swabs and mouth gargles. J Clin Microbiol 40:75-79

23. Bidmos FA, Neal KR, Oldfield NJ, Turner DP, Ala'Aldeen DA, Bayliss CD (2010) Rapid clonal expansion, persistence and clonal replacement of meningococcal carriage isolates in a 2008 university student cohort. J Clin Microbiol 49:506-512

24. Jounio U, Saukkoriipi A, Bratcher HB, Bloigu A, Juvonen R, Silvennoinen-Kassinen S et al (2012) Genotypic and phenotypic characterization of carriage and invasive disease isolates of Neisseria meningitidis in Finland. J Clin Microbiol 50:264-273

25. Drayß M, Claus H, Hubert K, Thiel K, Berger A, Sing A et al (2019) Asymptomatic carriage of Neisseria meningitidis, Haemophilus influenzae, Streptococcus pneumoniae, group a Streptococcus and Staphylococcus aureus among adults aged 65 years and older. PLoS One 14:e0212052

26. Miglietta A, Innocenti F, Pezzotti P, Riccobono E, Moriondo M, Pecile $P$ et al (2019) Carriage rates and risk factors during an outbreak of invasive meningococcal disease due to Neisseria meningitidis serogroup C ST-11 (cc11) in Tuscany, Italy: a crosssectional study. BMC Infect Dis 19:29

27. Memish ZA, Al-Tawfiq JA, Almasri M, Azhar EI, Yasir M, AlSaeed MS et al (2017) Neisseria meningitidis nasopharyngeal carriage during the Hajj: a cohort study evaluating the need for ciprofloxacin prophylaxis. Vaccine. 35:2473-2478

28. Tryfinopoulou K, Kesanopoulos K, Xirogianni A, Marmaras N, Papandreou A, Papaevangelou V et al (2016) Meningococcal carriage in military recruits and university students during the pre MenB vaccination era in Greece (2014-2015). PLoS One 11:e0167404

29. McMillan M, Walters L, Mark T, Lawrence A, Leong LEX, Sullivan T et al (2019) B part of it study: a longitudinal study to assess carriage of Neisseria meningitidis in first year university students in South Australia. Hum Vaccin Immunother 15:987-994

30. Maiden MC, Stuart JM, UK Meningococcal Carriage Group (2002) Carriage of serogroup $\mathrm{C}$ meningococci 1 year after meningococcal C conjugate polysaccharide vaccination. Lancet 359:1829-1831

31. Kristiansen PA, Ba AK, Ouédraogo AS, Sanou I, Ouédraogo R, Sangaré L et al (2014) Persistent low carriage of serogroup a Neisseria meningitidis two years after mass vaccination with the meningococcal conjugate vaccine, MenAfriVac. BMC Infect Dis 14:663

32. Read RC, Baxter D, Chadwick DR, Faust SN, Finn A, Gordon SB et al (2014) Effect of a quadrivalent meningococcal ACWY glycoconjugate or a serogroup B meningococcal vaccine on meningococcal carriage: an observer-blind, phase 3 randomised clinical trial. Lancet 384:2123-2131

33. Bundesministerium für Arbeit, Soziales, Gesundheit und Konsumentenschutz (2020) Impfplan Österreich 2020. https:// www.sozialministerium.at/dam/jcr:a0cf83df-7c20-48ac-b024e6015a45a753/200103_Impfplan_\%C3\%96sterreich_2020 pdfUA.pdf. Accessed 31 Mar 2020

34. Coch Gioia CA, Silva de Lemos AP, Outeiro Gorla MC, MendozaSassi RA, Ballester T, Von Groll A et al (2015) Detection of Neisseria meningitidis in asymptomatic carriers in a university hospital from Brazil. Rev Argent Microbiol 47:322-327

35. Gaunt PN, Lambert BE (1988) Single dose ciprofloxacin for the eradication of pharyngeal carriage of Neisseria meningitidis. J Antimicrob Chemother 21:489-496

36. Austrian Agency for Health and Food Safety (2019) National Reference Center for meningococcal disease. Annual Report 2018. https://www.ages.at/service/service-oeffentliche-gesundheit/ referenzzentralen/rz-meningokokken. Accessed 22 Oct 2019

37. De Wals P, Gilquin C, De Maeyer S, Bouckaert A, Noel A, Lechat MF et al (1983) Longitudinal study of asymptomatic meningococcal carriage in two Belgian populations of schoolchildren. J Inf Secur 6:147-156

38. MenAfriCar consortium (2016) Household transmission of Neisseria meningitidis in the African meningitis belt: a longitudinal cohort study. Lancet Glob Health 4:e989-e995

39. Krone M, Gray S, Abad R, Skoczyńska A, Stefanelli P, van der Ende A et al (2019) Increase of invasive meningococcal serogroup W disease in Europe, 2013 to 2017. Euro Surveill 24:1800245

40. Breakwell L, Whaley M, Khan UI, Bandy U, Alexander-Scott N, Dupont L et al (2018) Meningococcal carriage among a university student population - United States, 2015. Vaccine 36:29-35

41. Jeppesen CA, Snape MD, Robinson H, Gossger N, John TM, Voysey $\mathrm{M}$ et al (2015) Meningococcal carriage in adolescents in the United Kingdom to inform timing of an adolescent vaccination strategy. J Inf Secur 71:43-52

42. Kim HW, Lee S, Kwon D, Cha J, Ahn JG, Kim KH (2017) Characterization of oropharyngeal carriage isolates of Neisseria meningitidis in healthy Korean adolescents in 2015. J Korean Med Sci 32:1111-1117

43. Trotter CL, Gay NJ (2003) Analysis of longitudinal bacterial carriage studies accounting for sensitivity of swabbing: an application to Neisseria meningitidis. Epidemiol Infect 130:201-205

44. Manigart O, Okeakpu J, Odutola A, Jarju S, Foster-Nyarko E, Diallo K et al (2016) Alternative molecular methods for improved detection of meningococcal carriage and measurement of bacterial density. J Clin Microbiol 54:2743-2748

Publisher's note Springer Nature remains neutral with regard to jurisdictional claims in published maps and institutional affiliations. 